\title{
Impact of Sleep Quality on Cardiovascular Outcomes in Hemodialysis Patients: Results from the Frequent Hemodialysis Network Study
}

\author{
Mark Unruh $^{a}$ Manjula Kurella Tamura ${ }^{b}$ Brett Larive ${ }^{c}$ Anjay Rastogi ${ }^{d}$ \\ Sam James ${ }^{e}$ Brigitte Schiller ${ }^{b, f}$ Jennifer Gassman ${ }^{c}$ Christopher Chan ${ }^{g}$ \\ Robert Lockridge $^{\mathrm{h}} \quad$ Alan Kliger $^{\mathrm{i}}$

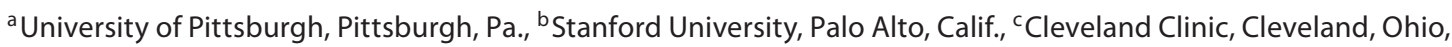

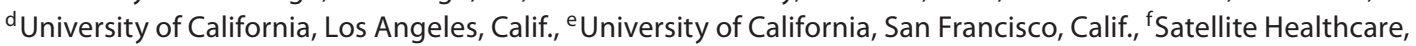

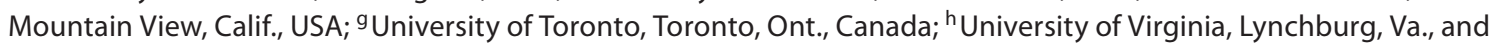 \\ 'Department of Medicine, Hospital of Saint Raphael, Yale University School of Medicine, New Haven, Conn., USA
}

\section{Key Words}

Hemodialysis · Sleep • Quality of life · Cognitive function •

Cardiac magnetic resonance imaging

\begin{abstract}
Background: Poor sleep quality is a common, persistent, and important problem to patients with end-stage renal disease (ESRD). This report examines whether sleep quality is associated with dialysis treatment factors and other modifiable clinical factors in a large group of hemodialysis (HD) patients. Methods: Cross-sectional analyses were conducted on baseline data collected from participants in the Frequent Hemodialysis Network trials. Sleep quality was measured using the Medical Outcomes Study Sleep Problems Index II (SPI II), a 9-item measure of sleep quality with higher scores reflecting poorer sleep quality. Results: The participants had an age of $51.2 \pm 13.6$ years, $61 \%$ were male, $38 \%$ were black, and $42 \%$ had diabetes. Higher pre-dialysis serum phosphorus (per $0.5 \mathrm{mg} / \mathrm{ml})(\mathrm{OR} 0.91 ; 95 \% \mathrm{Cl} 0.85,0.96)$ and depression (OR $0.16 ; 95 \% \mathrm{Cl} 0.10,0.25)$ were independently associated with decrements in sleep quality. There was also a difference in time to recovery from dialysis for the fourth
\end{abstract}

versus the first SPI II quartile (5.1 h; $\mathrm{p}<0.0001)$. Conclusion: These findings underscore the link between sleep and daytime function and suggest that improving sleep may provide an opportunity to improve outcomes in ESRD. Whether sleep problems may be improved by reduction of serum phosphorus or treatment of depression in the HD population merits further investigation.

Copyright $\odot 2011$ S. Karger AG, Basel

\section{Introduction}

Symptoms related to sleep and fatigue are common, persistent, and important to patients with end-stage renal disease (ESRD) [1-6]. In a systematic review of 59 studies evaluating the prevalence of various symptoms among patients on dialysis, several of the most common and severe symptoms were related to sleep and fatigue with fatigue/tiredness endorsed by an average of 71\%, disturbed sleep by $44 \%$, and restless legs by $30 \%$ [7]. In the ESRD population, complaints of sleep problems have been linked to disability days, healthcare utilization, and health-related quality of life (HRQOL) [8-11]. A number

\section{KARGER}

(c) 2011 S. Karger AG, Basel

Fax +41613061234

E-Mail karger@karger.ch

www.karger.com
Accessible online at: www.karger.com/ajn
Mark L. Unruh, MD, MS

University of Pittsburgh, Presbyterian Hospital C1111

200 Lothrop Street, Pittsburgh, PA 15213 (USA)

Tel. +1 4126472571

E-Mail unruh@pitt.edu 
of studies in the ESRD population have demonstrated strong associations between sleep quality and mental and physical well-being [8-13]. In a survey of 100 hemodialysis (HD) patients, $93 \%$ would do more frequent HD treatments to improve fatigue and $57 \%$ would do more frequent treatments to improve sleep [14]. This contrasts with $19 \%$ of patients willing to undergo more frequent dialysis in order to live up to 3 more years [14]. These findings underscore the profound impact of sleep on the experiences of patients with ESRD.

While self-reported sleep quality is important to ESRD patients, few modifiable factors have been identified to tailor effective treatments for sleep in this population. In cross-sectional studies, Merlino et al. [6] posited that insomnia in the dialysis population was related to older age, cigarette and alcohol use and neuropathy. Unruh et al. [10] demonstrated that poorer sleep quality was associated with younger age, black race, higher phosphate, and current smoking. Other reports have linked sleep quality to lower hemoglobin and depression [15]. The impact of dialysis dose and flux on sleep quality was tested as a prespecified quality of life outcome in the multi-center HEMO Study, a randomized clinical trial of HD dose and flux among patients receiving $3 \times$ weekly HD [16]. There was no significant effect of higher versus lower Kt/V urea on sleep quality, as assessed by the Kidney Disease Quality of Life-Long Form. High-flux HD had a small beneficial effect on sleep quality which did not reach significance after accounting for multiple comparisons [16]. Longitudinal analysis of HEMO Study participants demonstrated that approximately $30 \%$ experienced a clinically significant decline in sleep quality over the course of 3 years [17]. Since dialysis dose and flux did not substantially improve sleep quality, it is important to assess whether modifiable factors such as serum phosphate, depression, and tobacco use are associated with sleep quality in contemporary dialysis patients.

The Frequent Hemodialysis Network (FHN) Trials provide a unique opportunity to assess the relationship between sleep quality and nutritional markers, comorbidities, depression, anemia, bone mineral disorders, and central nervous system (CNS)-active medications. The FHN Trials consist of two randomized trials comparing more frequent $\mathrm{HD}$ schedules with conventional $3 \times$ weekly HD schedules for patients with ESRD. We also assess the extent to which poor sleep quality is associated with reduced HRQOL, more extensive left ventricular mass (LVM), as well as diminished cognitive performance in this contemporary cohort of prevalent HD patients.

Hyperphosphatemia, Depression and Poor Sleep Quality in HD Patients

\section{Subjects and Methods}

\section{Subjects}

The design, recruitment, and measurements of the FHN Trials have been previously published [18]. Briefly, we recruited participants with ESRD requiring maintenance $\mathrm{HD}$ from clinical sites in the USA and Canada for one of two trials: a trial of $6 \times$ per week in-center HD versus $3 \times$ per week in-center HD ('in-center Daily Trial') or a trial of $6 \times$ per week home nocturnal dialysis versus $3 \times$ per week in-center or home HD ('Nocturnal Trial'). The initial Nocturnal study protocol specified that Conventional arm patients receive $3 \times$ weekly in-center $H D$. Due to difficulties with recruitment, a revised protocol was adopted in which all of the last 72 participants were first trained in home $\mathrm{HD}$, and those patients randomized to the $3 \times$ arm received $H D$ at home rather than in a center. Major exclusion criteria included age $<13$ (Daily) or $<18$ (Nocturnal) years, inability to achieve a mean $\mathrm{eKt} / \mathrm{V}_{\text {urea }} \geq 1.0$ on two occasions, life expectancy $<6$ months, medical need for HD $>3 \times$ per week, history of poor adherence to $\mathrm{HD}$, medical conditions preventing cardiac magnetic resonance imaging, inability to communicate in English or Spanish, and anticipated kidney transplantation or relocation within the next 14 months. The study was reviewed by institutional review boards at each clinical center, and all participants signed informed consent. The FHN Trials collected HRQOL and sleep quality data using trained interviewers and computer-assisted telephone interviewing. For these analyses, we included individuals who were enrolled in either trial and completed baseline sleep questionnaires, whether or not they were randomized. We excluded individuals $<21$ years of age and those missing baseline sleep scores, resulting in an analytic cohort of 413 participants (fig. 1).

\section{Explanatory Variables and Covariates}

At enrollment, we recorded demographic and clinical characteristics, anthropometric measures, HD treatment parameters, laboratory measures and medication use for each study subject. Comorbid conditions were assessed using the modified Charlson Comorbidity Index [19]. Depressive symptoms was defined as a score $>15$ on the Beck Depression Inventory [20, 21]. Laboratory variables were measured pre-dialysis at laboratories affiliated with each clinical center.

We a priori selected five main categories of predictor variables: nutritional markers, comorbidities, anemia, markers of bone mineral disorders (calcium, phosphorus, and parathyroid hormone), and CNS-active medications. We characterized the weekly clearance of urea using standard $\mathrm{Kt} / \mathrm{V}\left(\mathrm{stdKt} / \mathrm{V}_{\text {urea }}\right)$, calculated as the average stdKt $/ \mathrm{V}_{\text {urea }}$ from two baseline kinetic modeling sessions, as previously described [22]. We selected two nutritional markers: body mass index (BMI) and serum albumin concentration. Body weight was measured immediately after a HD session. We selected three comorbidity markers: diabetes, the Charlson Comorbidity Index, and depression as measured by the Beck Depression Index. Use of the following CNS-active medications was recorded for each subject: benzodiazepines, antidepressants, anticonvulsants, $\mathrm{H}_{1}$-receptor antagonists, and opioids.

We also examined the relationship of sleep quality to HRQOL, cognitive functioning, and LVM. HRQOL was assessed in FHN using the SF-36, a questionnaire which has been used extensively in patients with ESRD [23]. From the SF-36, four domains were examined a priori: bodily pain is a 2 -item scale that evaluates the 


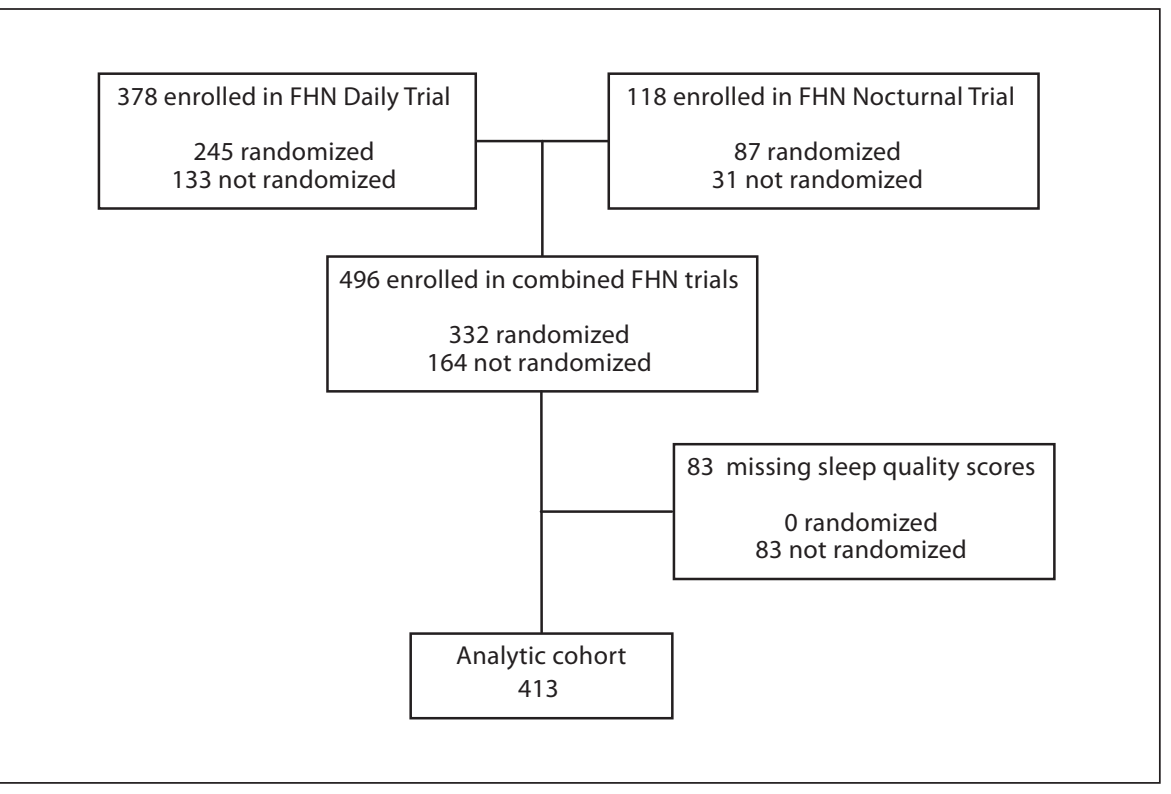

Fig. 1. Derivation of the analytic cohort.

perceived amount of pain experienced during the previous 4 weeks and the extent to which that pain interfered with normal work activities; vitality is a 4 -item scale that evaluates feelings of pep, energy, and fatigue; the RAND Physical Health Composite (PHC) and Mental Health Composite (MHC) were also examined as global measures of physical and mental well-being. Higher scores on each of these scales denote better HRQOL. In addition, we also assessed the time to recovery after dialysis which has been shown to be reliable and have construct validity in a HD cohort [24]. The composite scores were selected as a global test of quality of life. Since fatigue and pain have been related to sleep quality in the other chronic health conditions [25], these scales were selected to examine the strength of these relationships in the ESRD population. Trained study coordinators assessed cognitive function at enrollment with two cognitive tests. The Modified MiniMental State Exam (3MS) is a test of global cognitive function which includes an assessment of orientation, attention, calculation, language and short-term memory [26]. Scores range from 0 to 100 , lower scores indicate poorer cognitive function. The Trailmaking B (Trails B) test is a timed test of executive function, a cognitive domain not assessed with the 3MS [27]. Higher scores on the Trails B indicate poorer executive function. LVM was assessed using cine magnetic resonance imaging, which has been shown to be more accurate and less volume and operator dependent than $2 \mathrm{D}$ echocardiography with intra-observer reproducibility [28].

\section{Dependent Variable}

The Medical Outcomes Study (MOS) Sleep Problems Index (SPI) is a 12-item measure that includes items on sleep initiation and maintenance, sleep adequacy, daytime somnolence, and respiratory disturbance; participants are instructed to relate responses to sleep habits over the previous month [29,30]. The 9 items of the SPI are summed to obtain an overall SPI II score which has been shown to have good internal consistency and reli- ability, discriminative validity, and responsiveness to interventions [31-33]. A higher SPI II score reflects more extensive sleep problems.

\section{Statistical Analysis}

Baseline characteristics of the analytic sample were stratified according to trial and randomization status, and characterized using means $( \pm S D)$, medians (interquartile range, IQR) or frequencies (\%), as appropriate. We determined the association of each explanatory variable with SPI II quartile separately using logistic regression, expressed as an odds ratio (OR) and 95\% confidence interval (95\% CI). Variables which demonstrated nonlinear associations with sleep quality scores were divided into categories based on conventionally defined cut points (Beck Depression Index). Next, we determined the case mix-adjusted association of each explanatory variable after adjustment for age, gender, race, ESRD vintage, and diabetes. Because English fluency may influence the reporting of sleep quality score, we ran parallel analyses after excluding individuals whose primary language was not English. We also assessed whether associations might differ by FHN trial status (Daily vs. Nocturnal) or randomization results (randomized vs. not) through interaction tests. Finally, we developed hierarchical multivariable models selecting from variables significant at the $\mathrm{p}<0.1$ level in the case mix-adjusted analyses. Before developing these multivariable models, missing values were multiply imputed while incorporating auxiliary variables reflecting factors for baseline drop-out. The auxiliary variables were incorporated to better accommodate the assumption that missing data would be associated with the variables used for imputation. We used a linear discriminate model for imputation to minimize potential bias in the results for categorical variables [34]. Standard errors for the multivariable models corrected for the multiple imputation. We assessed collinearity between variables using variance inflation factor statistics. All analyses were conducted using SAS v9.1 (Cary, N.C., USA). 
Table 1. Subject characteristics during baseline for FHN patients with data for the MOS sleep scale

\begin{tabular}{|c|c|c|c|c|c|c|c|c|c|c|}
\hline \multirow[t]{2}{*}{ Variables } & \multirow[t]{2}{*}{$\mathrm{n}$} & \multirow{2}{*}{$\begin{array}{l}\text { All } \\
(n=413)\end{array}$} & \multicolumn{4}{|c|}{ Daily trial } & \multicolumn{4}{|c|}{ Nocturnal trial } \\
\hline & & & $\mathrm{n}$ & $\begin{array}{l}\text { randomized } \\
(\mathrm{n}=245)\end{array}$ & $\mathrm{n}$ & $\begin{array}{l}\text { enrolled only } \\
(n=63)\end{array}$ & $\mathrm{n}$ & $\begin{array}{l}\text { randomized } \\
(\mathrm{n}=87)\end{array}$ & $\mathrm{n}$ & $\begin{array}{l}\text { enrolled only } \\
(n=18)\end{array}$ \\
\hline Age, years & 413 & $51.2 \pm 13.6$ & 245 & $50.4 \pm 13.9$ & 63 & $52.3 \pm 13.0$ & 87 & $52.8 \pm 13.6$ & 18 & $49.8 \pm 12.3$ \\
\hline Female & 413 & $160(39 \%)$ & 245 & $94(38 \%)$ & 63 & $29(46 \%)$ & 87 & $30(34 \%)$ & 18 & $7(39 \%)$ \\
\hline Black race & 413 & $157(38 \%)$ & 245 & $102(42 \%)$ & 63 & $29(46 \%)$ & 87 & $23(26 \%)$ & 18 & $3(17 \%)$ \\
\hline Patient's primary language is not English & 413 & $68(16 \%)$ & 245 & $49(20 \%)$ & 63 & $7(11 \%)$ & 87 & $10(11 \%)$ & 18 & $2(11 \%)$ \\
\hline ESRD vintage, years* & 413 & $0.91 / 2.43 / 6.10$ & 245 & $1.71 / 3.64 / 7.24$ & 63 & $0.88 / 1.79 / 4.99$ & 87 & $0.30 / 0.91 / 3.54$ & 18 & $0.22 / 1.67 / 4.02$ \\
\hline Diabetes & 386 & $163(42 \%)$ & 245 & $100(41 \%)$ & 41 & $19(46 \%)$ & 87 & $37(43 \%)$ & 13 & $7(54 \%)$ \\
\hline Modified Charlson Comorbidity Index* & 386 & $0 / 1 / 3$ & 245 & $0 / 1 / 2$ & 41 & $0 / 2 / 4$ & 87 & $0 / 1 / 2$ & 13 & $0 / 1 / 4$ \\
\hline $\mathrm{BMI}^{*}$ & 413 & $22.2 / 26.1 / 32.9$ & 245 & $22.1 / 25.6 / 32.7$ & 63 & $21.8 / 25.7 / 34.0$ & 87 & $23.4 / 27.1 / 34.0$ & 18 & $21.6 / 25.0 / 27.9$ \\
\hline Albumin, g/dl & 399 & $3.92 \pm 0.43$ & 245 & $3.94 \pm 0.42$ & 52 & $3.89 \pm 0.38$ & 87 & $3.91 \pm 0.49$ & 15 & $3.83 \pm 0.55$ \\
\hline Pre-dialysis phosphorus, mg/dl & 399 & $5.79 \pm 1.69$ & 245 & $5.78 \pm 1.64$ & 52 & $5.67 \pm 1.88$ & 87 & $5.80 \pm 1.61$ & 15 & $6.41 \pm 2.29$ \\
\hline Hemoglobin, g/dl & 385 & $11.9 \pm 1.3$ & 244 & $11.9 \pm 1.3$ & 41 & $12.1 \pm 1.7$ & 87 & $11.8 \pm 1.1$ & 13 & $12.3 \pm 1.2$ \\
\hline Serum creatinine, $\mathrm{mg} / \mathrm{dl}$ & 399 & $9.86 \pm 3.08$ & 245 & $10.5 \pm 2.7$ & 52 & $8.87 \pm 3.94$ & 87 & $8.74 \pm 2.99$ & 15 & $8.53 \pm 2.90$ \\
\hline Parathyroid hormone, $\mathrm{mmol} / \mathrm{l}^{*}$ & 383 & $182 / 315 / 545$ & 242 & $183 / 313 / 611$ & 41 & $171 / 344 / 557$ & 87 & $194 / 322 / 443$ & 13 & $78.5 / 251 / 317$ \\
\hline Weekly standard Kt/V urea & 396 & $2.66 \pm 0.45$ & 245 & $2.60 \pm 0.33$ & 49 & $2.79 \pm 0.70$ & 87 & $2.73 \pm 0.43$ & 15 & $2.92 \pm 0.80$ \\
\hline Time to recovery, $\mathrm{h}^{*}$ & 407 & $1 / 3 / 6$ & 241 & $1 / 3 / 6$ & 62 & $1 / 3 / 12$ & 18 & $1 / 2 / 3$ & 18 & $1 / 2 / 3$ \\
\hline \multicolumn{11}{|l|}{ Pre-dialysis blood pressure, $\mathrm{mm} \mathrm{Hg}$} \\
\hline Systolic & 399 & $147 \pm 22$ & 245 & $147 \pm 20$ & 52 & $146 \pm 23$ & 87 & $152 \pm 20$ & 15 & $138 \pm 23$ \\
\hline Diastolic & 399 & $80.0 \pm 13.7$ & 245 & $79.9 \pm 13.1$ & 52 & $74.6 \pm 12.5$ & 87 & $83.8 \pm 14.5$ & 15 & $76.9 \pm 16.3$ \\
\hline \multicolumn{11}{|l|}{ Trailmaking B score $\geq 300 \mathrm{~s}$} \\
\hline (global cognitive impairment) & 380 & $46(12 \%)$ & 244 & $36(15 \%)$ & 39 & $7(18 \%)$ & 86 & $3(3 \%)$ & 11 & 0 \\
\hline Modified Mini-Mental Score & 384 & $87.9 \pm 9.5$ & 245 & $87.1 \pm 9.5$ & 41 & $87.0 \pm 12.6$ & 87 & $90.3 \pm 7.2$ & 11 & $89.6 \pm 8.7$ \\
\hline Left ventricular mass, $\mathrm{g}$ & 355 & $141 \pm 53$ & 245 & $140 \pm 54$ & 14 & $164 \pm 62$ & 87 & $137 \pm 46$ & 9 & $152 \pm 56$ \\
\hline Beck Depression Index $>15$ & 386 & $12(32 \%)$ & 231 & $80(31 \%)$ & 55 & $23(42 \%)$ & 83 & $24(29 \%)$ & 17 & $4(24 \%)$ \\
\hline Benzodiazepines & 390 & $53(14 \%)$ & 245 & $31(13 \%)$ & 45 & $7(16 \%)$ & 86 & $15(17 \%)$ & 14 & 0 \\
\hline Antidepressants & 390 & $68(17 \%)$ & 245 & $35(14 \%)$ & 45 & $10(22 \%)$ & 86 & $22(26 \%)$ & 14 & $1(7 \%)$ \\
\hline Opioids & 390 & $78(20 \%)$ & 245 & $42(17 \%)$ & 45 & $15(33 \%)$ & 86 & $18(21 \%)$ & 14 & $3(21 \%)$ \\
\hline Antiepileptics/anticonvulsants & 390 & $76(19 \%)$ & 245 & $50(20 \%)$ & 45 & $8(18 \%)$ & 86 & $17(20 \%)$ & 14 & $1(7 \%)$ \\
\hline Hypnotics, non-benzodiazepines & 390 & $36(9 \%)$ & 245 & $24(10 \%)$ & 45 & $5(11 \%)$ & 86 & $4(5 \%)$ & 14 & $3(21 \%)$ \\
\hline Antihistamines ( $\mathrm{H}_{1}$ receptors) & 390 & $95(24 \%)$ & 245 & $49(20 \%)$ & 45 & $10(22 \%)$ & 86 & $33(38 \%)$ & 14 & $3(21 \%)$ \\
\hline Sleep symptoms and problems scale & 413 & $36.2 \pm 20.3$ & 245 & $35.8 \pm 21.1$ & 63 & $42.1 \pm 20.4$ & 87 & $32.3 \pm 18.0$ & 18 & $39.7 \pm 16.1$ \\
\hline (quartiles) & 413 & $36.2 \pm 20.3$ & 245 & $35.8 \pm 21.1$ & 63 & $42.1 \pm 20.4$ & 87 & $32.3 \pm 18.0$ & 18 & $39.7 \pm 16.1$ \\
\hline$\leq 20$ & & $105(25 \%)$ & & $69(28 \%)$ & & $13(21 \%)$ & & $21(24 \%)$ & & $2(11 \%)$ \\
\hline$>20$ and $\leq 35.6$ & & $105(25 \%)$ & & $57(23 \%)$ & & $10(16 \%)$ & & $32(37 \%)$ & & $6(33 \%)$ \\
\hline$>35.6$ and $\leq 51.1$ & & $95(23 \%)$ & & $54(22 \%)$ & & $16(25 \%)$ & & $19(22 \%)$ & & $6(33 \%)$ \\
\hline$>51.1$ & & $108(26 \%)$ & & $65(27 \%)$ & & $24(38 \%)$ & & $15(17 \%)$ & & $4(22 \%)$ \\
\hline
\end{tabular}

* Results are shown as median and IQR. Otherwise data are presented as mean $\pm \mathrm{SD}$, or counts (\%), as appropriate.

\section{Results}

As shown in table 1 , there were 413 participants included in the analytic cohort, 308 participants enrolled in the in-center Daily Trial and 105 participants enrolled in the Nocturnal Trial. Participants included in the analytic cohort had a mean age of $51.2 \pm 13.6$ years, $61 \%$ were male, $38 \%$ were black, and $42 \%$ had diabetes (table 1 ). The median ESRD vintage was 2.4 (IQR 0.9-6.1) years. The mean SPI II score was $36.2 \pm 20.3$. There were no differences between the study sample and excluded FHN participants in age, gender, black race, ESRD vintage, modified Charlson's Index, diabetes, and English as a second language. There was a trend toward a difference in ESRD vintage among excluded participants (cohort median and IQ range $=2.43[0.91,6.10]$, excluded $=2.01[0.84,5.23]$; $\mathrm{p}=0.08$; Wilcoxon rank sums test).

Table 2 demonstrates the strength of association between sleep quality and both clinical and laboratory characteristics. Older age was significantly associated with better sleep and the relationship was strengthened after accounting for case-mix variables. The presence of diabetes, higher pre-dialysis phosphorus, and depression were associated with a lower likelihood of better sleep after case-mix adjustment. There was not a significant relationship between sleep quality and volume removal or parathyroid hormone concentration. Cardiovascular disease measures (LVM, SBP, DBP) and cognitive perfor- 
Table 2. Crude and case mix-adjusted associations of patient characteristics with a better sleep quality quartile

\begin{tabular}{|c|c|c|c|c|c|c|}
\hline Variables & $\mathrm{n}$ & Unadjusted & $\mathrm{p}$ & $\mathrm{n}$ & Adjusted for case mix $^{*}$ & $p$ \\
\hline Age (per decade) & 413 & $1.19(1.05,1.36)$ & 0.006 & 386 & $1.29(1.12,1.48)$ & $<0.0001$ \\
\hline Female & 413 & $0.85(0.59,1.21)$ & 0.36 & 386 & $0.89(0.61,1.29)$ & 0.54 \\
\hline Black race & 413 & $0.91(0.64,1.30)$ & 0.61 & 386 & $0.84(0.58,1.22)$ & 0.37 \\
\hline Patient's primary language is not English & 413 & $1.43(0.89,2.28)$ & 0.14 & 386 & $1.62(0.97,2.69)$ & 0.06 \\
\hline ESRD vintage, years & 413 & $1.01(0.98,1.03)$ & 0.73 & 386 & $1.00(0.97,1.03)$ & 0.85 \\
\hline Diabetes & 386 & $0.78(0.54,1.12)$ & 0.17 & 386 & $0.63(0.42,0.93)$ & 0.02 \\
\hline Modified Charlson Comorbidity Index (per unit) & 386 & $0.93(0.85,1.03)$ & 0.16 & 386 & $0.90(0.80,1.02)$ & 0.10 \\
\hline BMI $\left(\right.$ per $\left.\mathrm{kg} / \mathrm{m}^{2}\right)$ & 413 & $0.99(0.97,1.02)$ & 0.52 & 386 & $1.01(0.98,1.03)$ & 0.59 \\
\hline Albumin (per $0.1 \mathrm{~g} / \mathrm{dl}$ ) & 399 & $1.03(0.99,1.07)$ & 0.16 & 381 & $1.03(0.98,1.07)$ & 0.22 \\
\hline Pre-dialysis phosphorus (per $0.5 \mathrm{mg} / \mathrm{dl}$ ) & 399 & $0.91(0.86,0.96)$ & $<0.0001$ & 381 & $0.94(0.88,0.99)$ & 0.03 \\
\hline Hemoglobin (per g/dl) & 385 & $1.00(0.87,1.15)$ & 0.99 & 374 & $0.97(0.84,1.13)$ & 0.72 \\
\hline Serum creatinine (per mg/dl) & 399 & $0.98(0.93,1.04)$ & 0.55 & 381 & $1.02(0.95,1.09)$ & 0.64 \\
\hline Parathyroid hormone (per $50 \mathrm{mmol} / \mathrm{l}$ ) & 383 & $1.00(0.97,1.02)$ & 0.81 & 372 & $1.00(0.98,1.03)$ & 0.75 \\
\hline Weekly standard Kt/V urea (per 0.1 units) & 396 & $1.03(0.99,1.07)$ & 0.12 & 381 & $1.02(0.98,1.07)$ & 0.37 \\
\hline Residual renal function (per $\mathrm{ml} / \mathrm{min}$ ) & 396 & $1.05(0.94,1.16)$ & 0.40 & 381 & $1.00(0.89,1.13)$ & 0.98 \\
\hline \multicolumn{7}{|l|}{ Pre-dialysis blood pressure (per $10 \mathrm{~mm} \mathrm{Hg}$ ) } \\
\hline Systolic & 399 & $0.97(0.90,1.06)$ & 0.52 & 381 & $0.99(0.91,1.08)$ & 0.84 \\
\hline Diastolic & 399 & $0.93(0.82,1.06)$ & 0.28 & 381 & $0.95(0.83,1.09)$ & 0.50 \\
\hline Beck Depression Index $>15$ & 386 & $0.16(0.10,0.24)$ & $<0.0001$ & 363 & $0.17(0.11,0.26)$ & $<0.0001$ \\
\hline Modified Mini-Mental Score (per unit) & 384 & $0.99(0.98,1.01)$ & 0.52 & 379 & $1.00(0.98,1.02)$ & 0.95 \\
\hline Trailmaking B score $\geq 300 \mathrm{~s}$ & 380 & $0.75(0.43,1.31)$ & 0.32 & 375 & $0.62(0.35,1.10)$ & 0.10 \\
\hline Left ventricular mass (per $10 \mathrm{~g}$ ) & 355 & $0.97(0.94,1.01)$ & 0.12 & 355 & $0.97(0.93,1.01)$ & 0.20 \\
\hline Randomized vs. enrolled only & 413 & $1.74(1.12,2.70)$ & 0.14 & 386 & $1.66(0.98,2.80)$ & 0.06 \\
\hline Benzodiazepines & 390 & $0.47(0.28,0.80)$ & 0.005 & 382 & $0.51(0.30,0.89)$ & 0.02 \\
\hline Antidepressants & 390 & $0.53(0.33,0.86)$ & 0.009 & 382 & $0.55(0.34,0.91)$ & 0.02 \\
\hline Opioids & 390 & $0.50(0.32,0.79)$ & 0.002 & 382 & $0.46(0.28,0.73)$ & $<0.0001$ \\
\hline Antiepileptics/anticonvulsants & 390 & $0.62(0.40,0.98)$ & 0.04 & 382 & $0.58(0.37,0.93)$ & 0.02 \\
\hline Hypnotics, non-benzodiazepines & 390 & $0.42(0.23,0.80)$ & 0.007 & 382 & $0.40(0.21,0.78)$ & 0.006 \\
\hline Antihistamines $\left(\mathrm{H}_{1}\right.$ receptors $)$ & 390 & $0.83(0.55,1.25)$ & 0.36 & 382 & $0.86(0.56,1.33)$ & 0.46 \\
\hline
\end{tabular}

* Adjusted for case mix (age, gender, race, ESRD vintage and diabetes).

Table 3. Modifiable determinants independently associated with better sleep quality quartile

\begin{tabular}{|c|c|c|c|c|c|c|}
\hline \multirow[t]{2}{*}{ Variables } & \multicolumn{6}{|c|}{ Odds of association with better sleep quality } \\
\hline & model 1 & $\mathrm{p}$ & model 2 & $\mathrm{p}$ & model 3 & $\mathrm{p}$ \\
\hline Age (per decade) & $1.21(1.06,1.38)$ & 0.004 & $1.08(0.93,1.25)$ & 0.30 & $1.10(0.95,1.28)$ & 0.21 \\
\hline Female & $0.84(0.59,1.21)$ & 0.35 & $0.85(0.59,1.233)$ & 0.38 & $0.93(0.63,1.37)$ & 0.70 \\
\hline Black race & $0.93(0.65,1.33)$ & 0.70 & $0.85(0.58,1.23)$ & 0.39 & $0.84(0.57,1.23)$ & 0.37 \\
\hline ESRD vintage, years & $1.01(0.98,1.04)$ & 0.61 & $0.98(0.95,1.01)$ & 0.26 & $0.99(0.96,1.02)$ & 0.40 \\
\hline Randomized vs. enrolled only & $1.76(1.13,2.74)$ & 0.01 & $1.55(0.98,2.45)$ & 0.06 & $1.43(0.89,2.29)$ & 0.14 \\
\hline Diabetes & & & $0.71(0.46,1.09)$ & 0.19 & $0.73(0.47,1.13)$ & 0.16 \\
\hline Pre-dialysis phosphorus (per $0.5 \mathrm{mg} / \mathrm{dl}$ ) & & & $0.90(0.85,0.96)$ & $<0.001$ & $0.91(0.85,0.96)$ & 0.002 \\
\hline Beck Depression Index $>15$ (depression) & & & $0.16(0.10,0.25)$ & $<0.001$ & $0.16(0.10,0.25)$ & $<0.001$ \\
\hline Benzodiazepines & & & & & $0.94(0.50,1.75)$ & 0.84 \\
\hline Antidepressants & & & & & $0.81(0.47,1.39)$ & 0.45 \\
\hline Opioids & & & & & $0.64(0.38,1.08)$ & 0.01 \\
\hline Antiepileptics/anticonvulsants & & & & & $0.76(0.45,1.28)$ & 0.31 \\
\hline Hypnotics, non-benzodiazepines & & & & & $0.49(0.23,1.07)$ & 0.073 \\
\hline
\end{tabular}




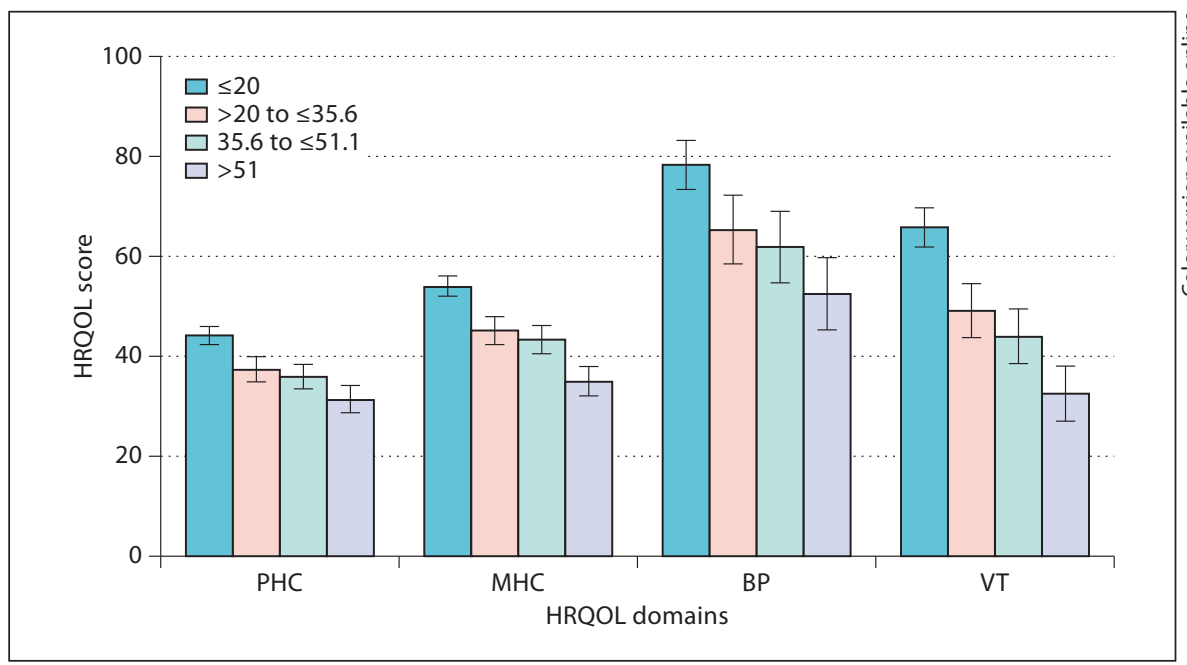

Fig. 2. Adjusted relationship between sleep quality quartile and self-reported HRQOL. The test for differences between the highest and lowest quartile was significant in each domain (Physical Health Component $(\mathrm{PHC})<0.0001$, Mental Health Component (MHC) $<0.0001$, Bodily Pain $(\mathrm{BP})<0.0001$, Vitality $(\mathrm{VT})<0.0001)$. The analysis was adjusted for age, sex, race, vintage and diabetes.

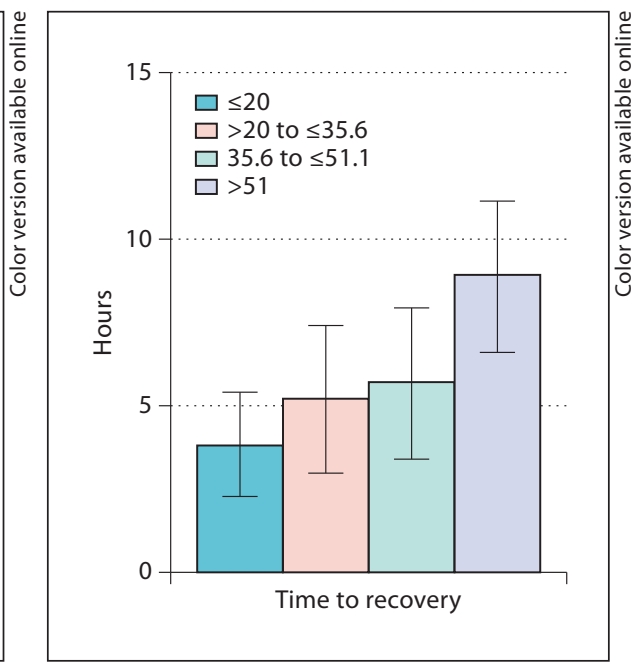

Fig. 3. Relationship between sleep quality and self-reported time to recovery. The test for differences between the highest and lowest quartile was significant $(\mathrm{p}<$ $0.0001)$. mance were not associated with sleep quality. The use of benzodiazepines, antidepressants, opioids, anticonvulsants, and non-benzodiazepine hypnotics were associated with lower sleep quality after adjusting for case mix.

\section{Multivariable Analyses}

Table 3 demonstrates the association of clinical factors with better sleep quality using a hierarchical logistic regression model. In model 1 using case-mix variables, older age and randomization were associated with better sleep quality. In model 2 , age and randomization were attenuated after controlling for diabetes, phosphorus and depression. In a multivariable adjusted model which included medications (model 3), pre-dialysis phosphorus and depression remained significantly associated with a decreased sleep quality (table 3). Whereas the strength of association between sleep quality and other covariates such as age, diabetes, randomized status, and psychoactive medications were attenuated.

\section{HRQOL and Time to Recovery}

Figure 2 demonstrates a strong graded relation between SPI II quartiles and pre-specified domains of HRQOL controlling for age, sex, race, vintage and diabetes. There were clinically significant differences in physical $(-12.8$ points; $\mathrm{p}<0.0001)$ and mental well-being $(-18.6$ points; $\mathrm{p}<0.0001)$ between the first versus the fourth quartile of sleep quality. The first versus the fourth quartile also had substantially worse scores in pain $(-25.7$; $p<$ $0.0001)$ and vitality $(-33.2 ; \mathrm{p}<0.0001)$. In figure 3 , there was a significant difference by sleep quality in time to recovery after dialysis after adjusting for case mix. In a sensitivity analysis, there was no substantial change in the strength of the relationship between sleep quality and HRQOL after adding depression to the adjusted model. Those with the poorest sleep quality had a recovery time of approximately $9 \mathrm{~h}$, or $5.1 \mathrm{~h}$ more than those with the best sleep quality; $\mathrm{p}<0.0001$.

\section{Discussion}

In a large cross-sectional study of HD patients from the USA and Canada, we found that sleep quality was significantly impaired. The SPI II average score in the FHN participants was 10 points higher (worse sleep quality) than scores from the general population [30]. Furthermore, over one quarter of our sample of HD patients reported sleep quality as poor as patients with restless legs or fibromyalgia $[32,33]$. We found that impairments in sleep quality were associated with clinically significant decrements in physical and mental well-being, vitality, and pain. We also found that worse sleep quality was significantly associated with longer recovery time from di- 
alysis. These findings highlight the marked impairment in sleep quality among contemporary HD patients and suggest that interventions to improve sleep could have a substantial positive impact on HRQOL in this population.

We found that a higher serum phosphate concentration was associated with decrements in self-reported sleep quality independent of parathyroid hormone, comorbidities or depression. This relationship between predialysis phosphorus and sleep quality is consistent with a small single-center study of a dialysis population using the SPI, which demonstrated that poor sleep quality was associated with male gender, coronary artery disease, and high serum phosphate level [5]. Higher phosphate was independently associated with decrements in sleep quality after accounting for age, race, tobacco use, benzodiazepines, and symptoms of restless legs in an incident dialysis population [10]. Higher serum phosphate has also been associated with impaired sleep efficiency in one study using self-reported sleep times [35]. While the FHN Trials did not assess symptoms of pruritus, in the Dialysis Outcomes and Practice Patterns Study reporting data from 18,801 HD patients, higher serum phosphate was associated with an increased likelihood of pruritus. In another single-center study, pruritus intensity was related to sleep quality [36]. Pruritus has also been associated with depression in the HD population [37]. However, in our study, the association of serum phosphate with sleep quality was independent of depressive symptoms. Thus, higher serum phosphate concentrations may be a marker of other conditions, such as pruritus or cardiovascular disease, which directly cause poor sleep quality.

This study demonstrates a strong association between depression and sleep quality using validated instruments to measure both sleep quality and depression. Devins et al. [38] demonstrated the association of depression with 'restless sleep' in a small sample of dialysis and kidney transplant patients. Some recent studies among patients with ESRD have demonstrated an association between poor sleep and depression, but have been limited by both small sample sizes and limited ability to adjust for potential confounders [39-42]. Other recent work in the Hungarian kidney transplant and dialysis population has demonstrated significant correlations between sleep and depressive symptoms $[43,44]$. Restless legs syndrome was associated with a high prevalence of depressive symptoms and diminished quality of life in a large sample of transplanted and waitlisted patients [44]. Their work has also demonstrated that patient-reported restless legs syndrome, insomnia and sleep apnea were independently as- sociated with quality of life [43]. This report extends these previous findings by demonstrating a relationship between sleep quality rather than self-reported sleep disorders. The FHN Trials may also provide a larger and more generalizable ESRD sample than these studies which examined only waitlisted HD patients. Nonetheless, this previous work provides a potential mechanism relating sleep quality to mood and HRQOL. The strong link between sleep quality and depression should also be interpreted in light of previous findings in the general population suggesting a bidirectional relationship between sleep quality and depression. In a community sample of 7,954 respondents, the risk of developing depression was much higher in patients with sleep complaints compared to those with no sleep complaints [45]. Insomnia has been shown to increase the risk for incident depression even after accounting for a previous history of depression [46] and sleep symptoms were associated with depression relapse [47]. The relationship between poor sleep quality and depression in the ESRD population is important since depression is treatable using either cognitive behavioral therapy [48] or antidepressant medications. In addition to sleep disorders which are thought to be widely prevalent in this population $[49,50]$, worsening sleep problems should heighten the providers' awareness for a potential depression.

The strengths of this report stem from the use of the FHN Trials which provide a large sample of patients from the USA and Canada with extensive data on comorbidity and near-perfect collection of sleep quality and HRQOL data using centrally administered surveys. The findings of this report should be interpreted in light of several limitations. This report is a cross-sectional study and is unable to discern the causal pathway for the observed relationships between poor sleep quality and both depression and hyperphosphatemia. Second, the FHN Trials uses the SPI from the Medical Outcomes Study to catalog sleep complaints in the study. The SPI II misses certain aspects of sleep such as nocturnal breathing, temperature, dreaming, and pain. However, the SPI is a widely used instrument for sleep quality and has been shown to respond to study interventions targeted toward improving sleep [51]. Third, the FHN study does not have the indication for medication use. While previous observational studies have shown that medications such as selective serotonin reuptake inhibitors or benzodiazepines were associated with poor sleep quality $[10,11]$, the interpretation of this association is difficult since the use of medications such as antidepressants or hypnotics may be due to complaints of poor sleep quality. Fourth, the FHN Study did not col- 
lect data on a history of diagnosis of sleep apnea. However, self-reported sleep quality is an important outcome in itself and there is discordance between patient-reported sleep quality and PSG findings among both the general population and the HD patients. It would be important in future HD studies to include both patient-reported and objective measures of sleep disorders using polysomnography or sleep apnea monitors.

Poor sleep is common and extremely bothersome to HD patients and among the primary reasons for considering more intensive HD therapies. Since sleep quality is strongly associated with quality of life and time to recovery from dialysis, these findings underscore the link between sleep and daytime function and support the position that improving sleep may provide an important opportunity to improve outcomes in ESRD. As previously noted, there is a need for treatment trials for poor sleep in ESRD [52]. We have identified several factors associated with poor sleep - elevated serum phosphate concentrations and depressive symptoms. It will be important in future work to understand the relationships between sleep quality, serum phosphorus control, depression, and HRQOL among those randomized to the frequent dialysis arm compared to the standard arm of FHN Daily. Future studies, including results of the FHN Trials, may provide insight into whether favorable modification of these factors might improve sleep quality and HRQOL.

\section{Acknowledgements}

This work was supported by grants from the National Institute of Diabetes and Digestive and Kidney Diseases (U01 DK03005) and M.U. (DK077785). The FHN investigator list is available in reference 37 .

\section{References}

$\checkmark 1$ Holley JL, Nespor S, Rault R: A comparison of reported sleep disorders in patients on chronic hemodialysis and continuous peritoneal dialysis. Am J Kidney Dis 1992;19: 156-161.

- Walker S, Fine A, Kryger MH: Sleep complaints are common in a dialysis unit. Am J Kidney Dis 1995;26:751-756.

- 3 De Vecchi A, Finazzi S, Padalino R, Santagostino T, Bottaro E, Roma E, Bossi R: Sleep disorders in peritoneal and haemodialysis patients as assessed by a self-administered questionnaire. Int J Artif Organs 2000;23: 237-242.

4 Hui DS, Wong TY, Ko FW, Li TS, Choy DK, Wong KK, Szeto CC, Lui SF, Li PK: Prevalence of sleep disturbances in Chinese patients with end-stage renal failure on continuous ambulatory peritoneal dialysis. Am J Kidney Dis 2000;36:783-788.

5 Unruh ML, Hartunian MG, Chapman MM, Jaber BL: Sleep quality and clinical correlates in patients on maintenance dialysis. Clin Nephrol 2003;59:280-288.

-6 Merlino G, Piani A, Dolso P, Adorati M, Cancelli I, Valente M, Gigli GL: Sleep disorders in patients with end-stage renal disease undergoing dialysis therapy. Nephrol Dial Transplant 2006;21:184-190.

7 Murtagh FE, Addington-Hall J, Higginson IJ: The prevalence of symptoms in end-stage renal disease: a systematic review. Adv Chronic Kidney Dis 2007;14:82-99.

-8 Hays RD, Kallich JD, Mapes DL, Coons SJ, Carter WB: Development of the kidney disease quality of life (KDQOL) instrument. Qual Life Res 1994;3:329-338.
\9 Iliescu EA, Coo H, McMurray MH, Meers CL, Quinn MM, Singer MA, Hopman WM: Quality of sleep and health-related quality of life in haemodialysis patients. Nephrol Dial Transplant 2003;18:126-132.

10 Unruh ML, Buysse DJ, Dew MA, Evans IV, Wu AW, Fink NE, Powe NR, Meyer KB: Choices for Healthy Outcomes in Caring for End-Stage Renal Disease Study: Sleep quality and its correlates in the first year of dialysis. Clin J Am Soc Nephrol 2006;1:802-810.

11 Elder SJ, Pisoni RL, Akizawa T, Fissell R, Andreucci VE, Fukuhara S, Kurokawa K, Rayner HC, Furniss AL, Port FK, Saran R: Sleep quality predicts quality of life and mortality risk in haemodialysis patients: results from the Dialysis Outcomes and Practice Patterns Study (DOPPS). Nephrol Dial Transplant 2008;23:998-1004.

$\$ 12$ Alvarez-Ude F, Alamo C, Fernaandez-Reyes MJ, Bravo B, Vicente E, Ferrer M, Alonso J, Badia X: Sleep complaints and perceived health status in patients in long-term hemodialysis. Nefrologia 1999;19:168-176.

13 Carmichael P, Popoola J, John I, Stevens PE, Carmichael AR: Assessment of quality of life in a single-centre dialysis population using the KDQOL-SF questionnaire. Qual Life Res 2000;9:195-205.

14 Ramkumar N, Beddhu S, Eggers P, Pappas LM, Cheung AK: Patient preferences for incenter intense hemodialysis. Hemodial Int 2005;9:281-295.

15 Pai MF, Hsu SP, Yang SY, Ho TI, Lai CF, Peng YS: Sleep disturbance in chronic hemodialysis patients: the impact of depression and anemia. Ren Fail 2007;29:673-677.
16 Unruh M, Benz R, Greene T, Yan G, Beddhu S, DeVita M, Dwyer JT, Kimmel PL, Kusek JW, Martin A, Rehm-McGillicuddy J, Teehan BP, Meyer KB, Group HS: Effects of hemodialysis dose and membrane flux on health-related quality of life in the HEMO Study. Kidney Int 2004;66:355-366.

17 Unruh ML, Newman AB, Larive B, Amanda Dew M, Miskulin DC, Greene T, Beddhu S, Rocco MV, Kusek JW, Meyer KB: The influence of age on changes in health-related quality of life over three years in a cohort undergoing hemodialysis. J Am Geriatr Soc 2008;56:1608-1617.

18 Suri RS, Garg AX, Chertow GM, Levin NW, Rocco MV, Greene T, Beck GJ, Gassman JJ, Eggers PW, Star RA, Ornt DB, Kliger AS: Frequent Hemodialysis Network randomized trials: study design. Kidney Int 2007;71: 349-359.

19 Beddhu S, Bruns FJ, Saul M, Seddon P, Zeidel ML: A simple comorbidity scale predicts clinical outcomes and costs in dialysis patients. Am J Med 2000;108:609-613.

20 Craven JL, Rodin GM, Littlefield C: The Beck Depression Inventory as a screening device for major depression in renal dialysis patients. Int J Psychiatry Med 1988;18:365374.

21 Watnick S, Wang PL, Demadura T, Ganzini L: Validation of two depression screening tools in dialysis patients. Am J Kidney Dis 2005;46:919-924.

-22 Greene T, Daugirdas JT, Depner TA, Gotch F, Kuhlman M: Solute clearances and fluid removal in the Frequent Hemodialysis Network trials. Am J Kidney Dis 2009;53:835844 
-23 DeOreo PB: Hemodialysis patient-assessed functional health status predicts continued survival, hospitalization, and dialysis-attendance compliance. Am J Kidney Dis 1997;30: 204-212.

-24 Lindsay RM, Heidenheim PA, Nesrallah G, Garg AX, Suri R: Minutes to recovery after a hemodialysis session: a simple health-related quality of life question that is reliable, valid, and sensitive to change. Clin J Am Soc Nephrol 2006;1:952-959.

-25 Beck SL, Dudley WN, Barsevick A: Pain, sleep disturbance, and fatigue in patients with cancer: using a mediation model to test a symptom cluster. Oncol Nurs Forum 2005; 32:542.

-26 Teng EL, Chui HC: The Modified MiniMental State (3MS) examination. J Clin Psychiatry 1987;48:314-318.

-27 Yeudall LT, Reddon JR, Gill DM, Stefanyk WO: Normative data for the Halstead-Reitan neuropsychological tests stratified by age and sex. J Clin Psychol 1987;43:346-367.

-28 Myerson SG, Bellenger NG, Pennell DJ: Assessment of left ventricular mass by cardiovascular magnetic resonance. Hypertension 2002;39:750-755

29 Hays R, Stewart A: Measuring Functioning and Well-Being: The Medical Outcomes Study Approach. Durham, Duke University Press, 1992.

-30 Hays RD, Martin SA, Sesti AM, Spritzer KL: Psychometric properties of the Medical Outcomes Study sleep measure. Sleep Med 2005; 6:41-44.

-31 Viala-Danten M, Martin S, Guillemin I, Hays RD: Evaluation of the reliability and validity of the Medical Outcomes Study sleep scale in patients with painful diabetic peripheral neuropathy during an international clinical trial. Health Qual Life Outcomes 2008;6:113.

>32 Allen RP, Kosinski M, Hill-Zabala CE, Calloway MO: Psychometric evaluation and tests of validity of the Medical Outcomes Study 12-item sleep scale (MOS sleep). Sleep Med 2009; 10:531-539.

-33 Cappelleri JC, Bushmakin AG, McDermott AM, Dukes E, Sadosky A, Petrie CD, Martin $S$ : Measurement properties of the Medical Outcomes Study sleep scale in patients with fibromyalgia. Sleep Med 2009;10:766-770.
34 Allison P: Imputation of categorical variables with PROC MI. Proc 30th Annual SAS Users Group International Conference, 2005. Cary, SAS institute Inc, 2005.

35 Koch BC, Nagtegaal JE, Hagen EC, van Dorp WT, Boringa JB, Kerkhof GA, Ter Wee PM Subjective sleep efficiency of hemodialysis patients. Clin Nephrol 2008;70:411-416.

36 Tessari G, Dalle Vedove C, Loschiavo C, Tessitore N, Rugiu C, Lupo A, Girolomoni G: The impact of pruritus on the quality of life of patients undergoing dialysis: a single-centre cohort study. J Nephrol 2009;22:241-248.

37 Yamamoto Y, Hayashino Y, Yamazaki S, Akiba T, Akizawa T, Asano Y, Saito A, Kurokawa K, Miyachi Y, Fukuhara S: Depressive symptoms predict the future risk of severe pruritus in haemodialysis patients: Japan dialysis outcomes and practice patterns study. Br J Dermatol 2009;161:384-389.

38 Devins GM, Edworthy SM, Paul LC, Mandin H, Seland TP, Klein G, Costello CG, Shapiro CM: Restless sleep, illness intrusiveness, and depressive symptoms in three chronic illness conditions: rheumatoid arthritis, end-stage renal disease, and multiple sclerosis. J Psychosom Res 1993;37:163-170.

39 Bilgic A, Akgul A, Sezer S, Arat Z, Ozdemir FN, Haberal M: Nutritional status and depression, sleep disorder, and quality of life in hemodialysis patients. J Ren Nutr 2007;17: 381-388.

40 Bornivelli C, Alivanis P, Giannikouris I, Arvanitis A, Choustoulakis I, Georgopoulou K, Karvouniaris N, Zervos A: Relation between insomnia mood disorders and clinical and biochemical parameters in patients undergoing chronic hemodialysis. J Nephrol 2008; 21(suppl 13):S78-S83.

41 Guney I, Biyik M, Yeksan M, Biyik Z, Atalay H, Solak Y, Selcuk NY, Tonbul HZ, Turk S Sleep quality and depression in peritoneal dialysis patients. Ren Fail 2008;30:10171022.

42 Paparrigopoulos T, Theleritis C, Tzavara C, Papadaki A: Sleep disturbance in haemodialysis patients is closely related to depression. Gen Hosp Psychiatry 2009;31:175-177.
43 Kovacs AZ, Molnar MZ, Szeifert L, Ambrus C, Molnar-Varga M, Szentkiralyi A, Mucsi I, Novak M: Sleep disorders, depressive symptoms and health-related quality of life - a cross-sectional comparison between kidney transplant recipients and waitlisted patients on maintenance dialysis. Nephrol Dial Transplant 2011;26:1058-1065.

44 Szentkiralyi A, Molnar MZ, Czira ME, Deak G, Lindner AV, Szeifert L, Torzsa P, Vamos EP, Zoller R, Mucsi I, Novak M: Association between restless legs syndrome and depression in patients with chronic kidney disease. J Psychosom Res 2009;67:173-180.

45 Ford DE, Kamerow DB: Epidemiologic study of sleep disturbances and psychiatric disorders. An opportunity for prevention? JAMA 1989;262:1479-1484.

46 Breslau N, Roth T, Rosenthal L, Andreski P: Sleep disturbance and psychiatric disorders: a longitudinal epidemiological study of young adults. Biol Psychiatry 1996;39:411418.

-47 Buysse DJ, Tu XM, Cherry CR, Begley AE, Kowalski J, Kupfer DJ, Frank E: Pretreatment REM sleep and subjective sleep quality distinguish depressed psychotherapy remitters and non-remitters. Biol Psychiatry 1999; 45:205-213

48 Duarte PS, Miyazaki MC, Blay SL, Sesso R: Cognitive-behavioral group therapy is an effective treatment for major depression in hemodialysis patients. Kidney Int 2009;76: 414-421.

49 Perl J, Unruh ML, Chan CT: Sleep disorders in end-stage renal disease: 'Markers of inadequate dialysis'? Kidney Int 2006;70:16871693.

50 Unruh M: Sleep in chronic kidney disease. Prim Psychiatry 2008;15:57-63.

51 Ferini-Strambi L, Aarskog D, Partinen M, Chaudhuri KR, Sohr M, Verri D, Albrecht S: Effect of pramipexole on RLS symptoms and sleep: a randomized, double-blind, placebocontrolled trial. Sleep Med 2008;9:874-881.

52 Novak M, Shapiro CM, Mendelssohn D, Mucsi I: Diagnosis and management of insomnia in dialysis patients. Semin Dial 2006; 19:25-31. 